\title{
US-Guided Fine-Needle Aspiration Cytology of Para- Aortic Lymph Nods Metastasis in Uterine Cervical Cancer: Accuracy and Impact on Clinical Decision Making
}

Junping Liu

Department of Ultrasound $\varangle$ Cancer Hospital of the University of Chinese Academy of Sciences $₫$ Zhejiang Cancer Hospital区

Xin Liu

Department of Ultrasound $₫$ Cancer Hospital of the University of Chinese Academy of Sciences $\$ Zhejiang Cancer Hospital区

\section{Zhengying Guo}

Department of Pathology $\llbracket$ Cancer Hospital of the University of Chinese Academy of Sciences $\bigotimes$ Zhejiang Cancer Hospital】

\section{Xiaojuan Lv}

Department of Gynecologic Oncology $\llbracket$ Cancer Hospital of the University of Chinese Academy of Sciences \Zhejiang Cancer Hospital『

\section{Weimin Mao}

Zhejiang Key Laboratory of the Diagnosis \& Treatment Technology on Thoracic Oncology

\section{Dong Xu}

Department of Ultrasound $₫$ Cancer Hospital of the University of Chinese Academy of Sciences $\ Z$ Zhejiang Cancer Hospital区

\section{Lijing Wang ( $\nabla$ wanglj844@zjcc.org.cn )}

Department of Ultrasound $₫$ Cancer Hospital of the University of Chinese Academy of Sciences $\$ Zhejiang Cancer Hospital区

\section{Research Article}

Keywords: cervical cancer, para-aortic lymph node metastases, fine needle aspiration cytology, diagnosis accuracy, ultrasound

Posted Date: April 22nd, 2021

DOI: https://doi.org/10.21203/rs.3.rs-395793/v1

License: (c) (i) This work is licensed under a Creative Commons Attribution 4.0 International License. Read Full License 


\section{Abstract}

Objective: To investigate whether ultrasound guided fine-needle aspiration cytology(US-FNAC) is an effective technique for diagnosing para-aortic lymph nodes(PALNs) metastasis in uterine cervical cancer and access the impact on clinical therapeutic decision.

Methods: We retrospectively reviewed the clinical data of 92 patients with PALN enlargement in cervical cancer between 2010 and 2018. The US-FNAC cytological results were classified by the same experienced cellular pathologists. The diagnostic indicators were calculated according to biopsy, imaging and clinical follow-up results. Univariate and multivariate analysis was used to analyze the differences of influencing factors. The effect of US-FNAC on clinical decision making was evaluated.

Results: The results of cytological diagnosis by US-FNAC were categorized as malignancy $(n=62 ; 67.4 \%)$, suspicious malignancy $(n=11 ; 12.0 \%)$, undetermined $(n=5 ; 5.4 \%)$, benign $(n=10 ; 10.9 \%)$, and inadequacy $(n=4 ; 4.3 \%)$. The satisfactory biopsy samples were obtained from $95.7 \%$ of PLANs (88/92). The sensitivity, specificity, PPV, NPV and accuracy of FNAC in distinguishing benign from malignant were $90.1 \%(95 \% \mathrm{Cl}: 0.809-0.953), 100 \%$ (95\%Cl:0.561-1), $100 \%(95 \% \mathrm{Cl}: 0.938-1), 46.7 \%(95 \% \mathrm{Cl}: 0.223-0.726)$ and $90.9 \%(95 \% \mathrm{Cl}: 0.848-0.970)$ respectively. Univariate analysis indicated that experience of the puncture physicians(radiologists) was significant differences between the correct diagnosis group and wrong diagnosis group $(P<0.05)$; the experience was confirmed as independent predictor of diagnostic accuracy by multivariate analysis $(\mathrm{p}=0.031, \mathrm{OR}=0.077,95 \% \mathrm{Cl}: 0.354-0.919)$. All patients tolerated the US-FNAC procedure well and only nine patients presented slight abdominal discomfort. Through US-FNAC technique, the therapeutic methods of 74 patients (80.4\%) were subjected to affect.

Conclusions: US-FNAC is a relatively safe and effective examination technique for enlarged para-aortic lymph nodes, which can be considered as a routine examination before treatment of cervical cancer to guide clinical decision-making.

\section{Key Findings}

1区The sensitivity, specificity, and accuracy of US-FNAC were $90.1 \%(95 \% \mathrm{Cl}: 0.809-0.953), 100 \%(95 \% \mathrm{Cl}: 0.561-1)$, and $90.9 \%(95 \% \mathrm{Cl}: 0.848-0.970)$ respectively.

$2 \otimes$ The experience of operators was confirmed as independent predictor of diagnostic accuracy by multivariate analysis $(p=0.031, \mathrm{OR}=0.077,95 \% \mathrm{Cl}: 0.354-0.919)$.

$3 \otimes$ The US-FNAC results influenced clinical therapeutic decision of $80.4 \%$ patients.

\section{Summary}

US-FNAC is a relatively safe and effective examination technique for enlarged PALNs, which can be considered as a routine examination before treatment of cervical cancer to guide clinical decision-making.

\section{Introduction}

Cervical cancer is the most common female genital tract malignant tumor and seriously endangers women's health and life. It is generally recognized that PALN metastasis involvement is a unfavorable prognostic indicator 
in cervical cancer patients, modifying treatment plan and associating with overall survival $(1,2)$. Thus, it is essential to accurately evaluate the PALN metastasis status .

However, confirmation of PALN metastases still presents a clinical problem. Computed tomography (CT) and magnetic resonance (MR) imaging identify retroperitoneal metastatic lymph nodes in basis of short-axis diameter(3). The weakness of the size-based criterion is that metastasis of normal size is easily missed (False negative) and enlarged reactive lymph nodes are misdiagnosed as metastases (False positive). Therefore, the investigators studied other diagnostic indicators, concerning contrast material used in the lymph nodes(4) and functional imaging method such as DW-MR(5) imaging and PET/CT(5-8). Some researchers demonstrated that DWI-MRI is high sensitive and PET/CT is the most specific and clinically relevant in detecting para-aortic lymphadenopathy from cervical cancer. However, PET/CT is expensive and unavailable in many districts, especially in developing countries. In addition, PET/CT may easily result in some unnecessary surgery and radiation in view of relatively high false-positive for predicting lymph node metastases(8).

Compared with the above non-invasive imaging methods, surgical biopsy is the gold standard for lymph node diagnosis. Lymphadenectomy has high risk and easily results in blood loss, prolonged hospitalization, postoperative adhesion formation(9). In order to avoid these complications and obtain pathological confirmation, more and more oncologists adopt and approve percutaneous image-guided biopsy technique that is routinely used for differential diagnosis, clinical staging, biological analysis and therapeutic strategy. Although CT-guided biopsy has high spatial resolution and visibility to achieve relatively satisfactory samples, the presence of nonportability, ionizing radiation, and high operating cost and time obviously hinder its clinical application(10,11).

Interestingly, US-FNAC is obtaining a important impact owing to real-time precise visualization and tracking, especially when there are vital structures adjacent to target lesion, including abdominal aorta, inferior vena cava, and gastrointestinal tract(12-14). However, US-FNAC remains underused for accessing the PALN metastasis status. In our department, interventional ultrasound has been carried out for nearly twenty years and US-FNAC is accepted as a first-line diagnostic technique for cervical cancer lymph node metastasis. The objective of this study was to investigate diagnostic accuracy of US-FNAC of enlarged PALNs in cervical cancers, emphasizing influencing factors and impact on treatment strategies.

\section{Materials And Methods}

2.1 Patients This study was approved by the Ethics Committee of Zhejiang Cancer Hospital and were in accordance with the ethical standards of the 1964 Helsinki Declaration. The need for informed consent from all patients was waived due to the study's retrospective. We used key words "retroperitoneal, " "para-aortic lymph node, " "biopsy, " and "aspiration" in a interventional ultrasound database between January 1, 2010 and December 30,2018. Figure 1 shows the workflow for the inclusion and exclusion of cases. According to the inclusion criteria, 240 cases were excluded from this study due to different conditions. Consequently, we consecutively enrolled 92 patients with PALNs from cervical cancer who were referred to our department for USFNAC by gynecological oncologists who required pathological results to guide the treatment of patients.

2.2 Ultrasound examination Before performing US-FNAC, each patient underwent a detailed ultrasound examination to determine the feasibility of FNA. The size, number, location, and characteristics of the affected lymph nodes were assessed using GE-E9₫5-16MHz囚and Esaote MyLab Twice (4-12MHz). The locations of the PLANs to be punctured were divided into four regions, levels $\mathbb{Z}-\mathbb{Z}$, depending on the anatomical structure(2)(Figure 
2). The size of nodules was measured in the central section of the cross-sectional image, taking the diameter perpendicular to the abdominal aorta as the length. The lymph nodes with safe puncture path and the most suspicious morphology were considered as suitable puncture targets. During undergoing US-FNAC, necrotic areas should be avoided as much as possible. In order to avoid damaging vessels in the puncture path, color Doppler was used to confirm the safest puncture path.

2.3 US-FNAC procedures Before puncture, all patients performed coagulation status screening (the international standardized ratio was less than 1.6, prothrombin time $<15 \mathrm{~s}$, partial thromboplastin time $<45 \mathrm{~s}$, platelets $>$ 80,000 per $\mathrm{mm}^{3}$.) , and provided informed consent for the US-FNAC procedure. The punctures were carried out by 5 radiologists; all were familiar with the FNAC process. The site of puncture was marked on the skin and the area was cleaned with povidone-iodine solution, and Local anesthesia was administered with $2 \%$ lidocaine. The USFNAC was performed a 20-23 gauge needle(Hakko Co, Japan) attached to an empty 5-mL plastic syringe, using negative suction with at least 1 pass(Figure 3). All operators used free hand technique (without biopsy attachment). The presence of on-site cytology evaluation does not be performed in our department. Patients were required in the hospital and were monitored for vital signs and complications.

Cellular material was then expelled onto a glass slide and the slides were immediately immersed in $95 \%$ alcohol. In some cases, cell block was carried out. The specimens were sent to the cytology department. The sample were stained using the haematoxylin and eosin staining method, which is the most commonly used for cytochemical staining, changes the refractive index of the cell's fine structure by color. The final cytology diagnosis were made by the same cellular pathologists with more than 10 years of experience.

2.4 FNAC results categorization and Final diagnosis. The FNAC results were classified into the following groups: unsatisfactory/nondiagnostic material for diagnosis(i, inadequate group), benign or reactive change(ii, benign group), atypical cells present but indeterminate for benign and malignancy(iii, undetermined group), suspicious for malignancy(iv, suspicious group), and positive for malignancy(v, malignant group). An unsatisfactory/nondiagnostic result(i) was defined as a sample consisting only of blood component or acellular specimen. When mature lymphocytes in morphology or linear colloid-like substance(foreign body granuloma) are obtained from aspirated nodules, benign results(ii) are reported. The undetermined results(iii) was defined as a sample consisting exclusively of a small amount of atypical cell components, but is not conclusive for benign and malignancy. When cytology diagnosis was reported as suspected malignant or unequivocally malignant, we defined the diagnosis as suspicious for malignancy(iv) or positive for malignancy(v). The unsatisfactory/nondiagnostic results(i) were defined as inadequate sample, while the remaining results were adequate/satisfactory specimen. Final diagnoses were determined in cases of (i,ii,iii) by repeat biopsy,surgery,imaging,clinical indicators,and clinical follow-up analyses(15).

2.5 Data Statistical Analyses. The cases of malignant(iv,v) were considered true-positive diagnosis. If the cases of (i,ii,iii) presented shrinkage, disappearance, or stationary condition about a period of 12-24 months without specific treatment by follow-up imaging, or if subsequent rebiopsy or surgery indicated a benign pathologic result, we defined cases of (i,ii,iii)as a true-negative result. A false-negative result was defined as (i,ii,iii) for which nodes were obviously enlarged by follow-up imaging or if the malignant results were confirmed by surgery and rebiopsy or if clinical data highly suggested malignant. We consided false-negative result as incorrect diagnosis. The evaluation index of diagnostic test involved specificity, sensitivity, negative predictive value, positive predictive value and accuracy. These parameters were calculated on the basis of the previous definition. 
Continuous variables were expressed as means \pm standard deviation, and the analysis was performed using ANOVA; categorical variables were presented on the basis of frequency and percentage, and the analysis was performed using a chi-square test or a Fisher exact test. Univariate analysis (chi-square test or a Fisher exact test) was performed to investigate the relationships among experience,age, body mass index, nodal size, nodal number, lesion location, needle size, cell block, nature and period. Multivariate logistic regression analysis was used to decide variables independently concerning the diagnostic yield of sampling. $\mathrm{P}<0.05$ for the difference was statistically significant. All statistical analysis uses SPSS statistical analysis software(version 21.0 for Windows; SPSS, Chicago, IL, USA).

\section{Results}

3.1 Clinical general data. There were 24 cervical cancer patients among these excluded cases(Figure 1). In sixteen of the 24 cases, the enlarged lymph nodes that can be showed in CT imaging cannot be visualized by conventional ultrasound. In five of the 24 cases in whom size of nodes were small $(<10 \mathrm{~mm})$, the US-FNAC operations were abandoned. There were no safe access path for puncture to help avoid injury to major vessels in three patients. Of the 92 cases, the age ranged from 25 to 80 years old (means:51.5 $\pm 10.8 \mathrm{y}$ ). The average size of nodes ranged from $11 \mathrm{~mm}$ to $28 \mathrm{~mm}$ (average: $17.1 \pm 4.0 \mathrm{~mm}$ ). In the 92 cases, they ranged in the body mass index

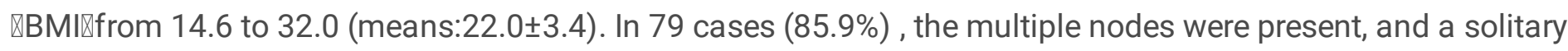
node was present in thirteen cases(14.1\%). $20 \mathrm{G}$ and $21 \mathrm{G}$ fine needles were used to perform the US-FNAC procedure in 60 cases. The biopsies was performed with $22 \mathrm{G}$ and $23 \mathrm{G}$ needle using suction in 32 cases.

The anatomical grouping of PLANs are divided into left and right distribution by abdominal aorta. The inferior mesenteric artery is taken as the boundary level line and divided into upper and lower region (Figure 2). There

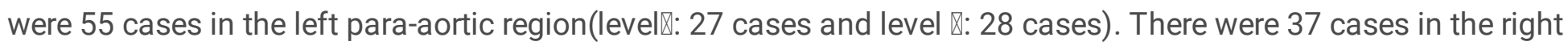
para-aortic region(level §: 29 cases and level $₫: 8$ cases)(Table 1).

3.2 Cytological findings and Final diagnosis. The results of cytological diagnosis by the biopsies were as follows: malignant( $n=62 ; 67.4 \%)$, suspicious $(n=11 ; 12.0 \%)$, undetermined $(n=5 ; 5.4 \%)$, benign $(n=10 ; 10.9 \%)$, and unsatisfactory $(n=4 ; 4.3 \%)$. The unsatisfactory materials were provided in 4 cases $(4.3 \%)$, but 5 cases belonged to undetermined group. In 2 undetermined cases and one inadequate case, a repeat US-FNAC was performed, which provided sufficient sample for the diagnosis of squamous cell carcinoma. The remaining 3 undetermined cases and 2 inadequate cases were confirmed as malignant by clinical follow-up and imaging for final diagnosis. However, the final benign diagnosis was established in one inadequate cases due to obvious shrinkage by four months follow-up. Therefore囚among 9 sample, there were 8 malignant(false-negative) and 1 benign cases(truenegative) in final diagnosis(Table 2).

Ten cases were reported as benign diagnosis(Figure 4a). Three cases of FNAC benign results were performed by subsequent surgical resections for therapeutic and diagnostic purposes and the definitive malignant diagnosis was rendered. The final diagnosis of the remaining 7 benign FNAC results were considered as benign diagnosis by clinical follow-up and imaging. There was no a false-positive result in the study. Therefore $\searrow$ final diagnosis in 10 benign sample consisted of 3 malignant(false-negative) and 7 benign cases(true-negative).

Eighty eight (95.7\%) biopsy materials were regarded as satisfactory sample by the more than 10 experienced cytopathologist. Of these 73 FNAC malignant specimens, 62 were considered as an unambiguous malignant diagnosis, including 5 cases of adenocarcinoma(Figure $4 b$ ), 39 cases of squamous cell carcinoma(Figure $4 c$ ), 
and 18 cases undifferentiated metastatic carcinoma(Figure 4d), while 11 were reported as a probable metastatic carcinoma diagnosis. According to the definition(iv,v),the 73 cases were all regarded as malignant cases in final diagnosis(true-positive).

3.3 Accuracy of US-FNAC. Due to different definitions of false negative and true negative that previous researchers have adopt for assessing diagnostic accuracy(16), we used the following analysis methods to present our data in this study. When we excluded the four inadequate cases in our analyses and regarded undetermined specimens as negative cytologic results. The values of the true positive, false positive, false negative and true negative were 73, 0, 8 and 7 respectively. The sensitivity, specificity, PPV, NPV and accuracy of FNAC in distinguishing benign from malignant were $90.1 \%(95 \% \mathrm{Cl}: 0.809-0.953), 100 \%(95 \% \mathrm{Cl}: 0.561-1), 100 \%$ (95\%Cl:0.938-1), 46.7\%(95\%Cl:0.223-0.726) and 90.9\%(95\%Cl:0.848-0.970) respectively. If the five cases with undetermined cytologic diagnosis were excluded, the values of the true positive, false positive, false negative and true negative were 73, 0, 3 and 7 respectively. The sensitivity,specificity,PPV,NPV and accuracy of FNAC in distinguishing benign from malignant were $96.1 \%(95 \% \mathrm{Cl}: 0.881-0.990), 100 \%(95 \% \mathrm{Cl}: 0.561-1), 100 \%(95 \% \mathrm{Cl}: 0.938-$ 1),70.0\%(95\% Cl:0.354-0.919) and $96.4 \%(95 \% \mathrm{Cl}: 0.923-1)$ respectively. There were no significant difference between the two methods (Table 3 ).

3.4 Factors influencing diagnostic accuracy. Table 4 summarized and compared the correct diagnosis group $(n=81)$ and incorrect diagnosis group (false negative cases, $n=11$ ) concerning variables affecting diagnostic accuracy. Experience refers to the years of engaging in interventional ultrasound. Univariate analysis revealed that the diagnostic yield of US-FNAC was significantly related to experience $(\leq 5 y$ vs. $>5 y)(p=0.008)$, but not with age, body mass index ( $\leq 24$ vs. $>24$ ), nodal size $(\leq 15 \mathrm{~mm}$ vs. $>15 \mathrm{~mm}$ ), nodal number(multiple vs. solitary), lesion location(left paraaortic vs. Right paraaortic), needle size( $\leq 21 \mathrm{G} v \mathrm{~s}$. $\geq 22 \mathrm{G})$, cell block(yes vs. no), nature (malignant vs. benign) and period (2010-2015 vs. 2016-2018) ( $p>0.05$ ). Furthmore, on the multivariate logistic regression analysis, it was showed that more experienced operators yielded correct diagnosis more easily $(p=0.031,0 \mathrm{R}=0.077,95 \% \mathrm{Cl}: 0.354-0.919)$ (Table 4).

3.5 Complications. All patients tolerated the US-FNAC procedure well. Major complications were not occurred during the US-FNAC procedures, such as bleeding, perforation and infection. Nine patients presented slight abdominal discomfort which was relieved after one hour of clinical observation. No further clinical treatment was required.

3.6 Impact of US-FNAC on clinical decision making. Radiotherapy, chemotherapy, and surgery (laparoscopy or laparotomy) should be used in the patients with PALN metastases from cervical cancer. After US-FNAC, 73 patients of cytological malignant patients, of which 59 patients were treated with PLAN radiotherapy and systemic chemotherapy and 9 patients only received chemotherapy. The size of PLANs were significantly reduced after treatment. Surgery was performed in 5 patients. Of the 10 patients with benign cytological diagnosis, 3 patients who was highly suggestive of malignancy by PET/CT imaging, underwent surgical biopsy and were diagnosed as metastatic squamous cell carcinoma. The remaining 7 patients required only observation. After 1224 months of follow-up, 3 patients experienced reduction in size of PLANs, while was stable in 4 patients. Of the 4 patients with unsatisfactory samples and 5 undetermined cases, 5 patients were treated with PLAN radiotherapy and systemic chemotherapy on the basis of PET/CT imaging and 3 patients received the same therapy according to secondary US-FNAC result, and there was significantly reduction in the size of PLANs. One patient who was not suggestive of malignancy by PET/CT imaging, was selected for clinical follow-up and then PLAN was shortened 
after 4 months. Therefore,through US-FNAC technique, the therapeutic methods of 74 patients $(80.4 \%)$ were subjected to affect.

\section{Discussion}

In the current study, our results indicate that FNAC is useful as a technique by sonographic guidance for PLANs in cervical cancer, whose diagnostic accuracy is $90.9 \%$. One of advantages is that the needle tip is displayed in real time throughout the procedure and the needle tip always remains within the lesion during sampling, which may minimize the sample being contaminated with blood and extraneous tissue (movie1). Another advantage of ultrasound guidance is that the manipulator can regulate toward almost any anatomical plane by angling and rotating the transducer to avoid visible blood vessels and important organs. When the needle tip will no longer be seen with US during procedure, we can use gently shaking the puncture needle to track the needle tip position(movie2). However, among 24 excluded cases in our study, 16 US-FNAC operations were abandoned because the enlarged lymph nodes that can be showed in CT imaging cannot be visualized by conventional ultrasound. The possible explanations are small nodules, intestinal gas interference and flake growth of PLANs.

All the patients endured the US-FNAC procedure and there was no apparent evidence of complications. Perforation that is a very worrying complication in the process of abdominal FNAC has not occurred, so antibiotics were not used in patients after biopsy. The finding was also reported by earlier studies(12-14) . In fact, it is difficult to distinguish bowel from mesentery or omentum after transducer compression and so we made no more effort to follow whether there were bowels in the puncture path. On the whole, the US-FNAC procedure is safe under clinical observation.

Similar to EUS-FNA technique(17) 囚the percutaneous US-FNAC for PLANs is thought to be highly challenging technique that is difficult to master and might make an great effort before the operator achieves an acceptable diagnostic yield. That is, the success rate of US-FNAC is directly associated with the radiologists experience. Experience refers to the years of engaging in interventional ultrasound. The current research found that of the 11 incorrect diagnoses, 9 cases were less than 3 years of experience. It was generally showed that the practitioner probably require a long learning curve to get the skills that contain obtaining a good view of the target node, selecting a suitable size needle and an appropriate FNAC operating technique. Therefore, we advise that the complicated technique should only be performed by those who have been undergone professional biopsy course training. The following research will focus on describing the learning curve of FNAC as a reference for training.

Our finding indicated that the US-FNAC approach influenced clinical decision making of 74 patients (80.4\%). If patients are found to have PLAN enlargement, paraaortic irradiation should be given only when the patient has pathological evidence of PALN metastasis, otherwise spinal cord inhibition and intestinal toxicity injury will be brought. In our study, it is encouraging that US-FNAC results was not false positive, which was also reported by Fisher et al(13). If these patients with cytologically benign PALN can be considered for conservative observation, we should integrate clinical and imaging data(15). Three patients with benign cytological diagnosis were diagnosed as metastatic squamous cell carcinoma by surgical biopsy in our study. One possible interpretation for the finding is that the needle passes may not sample the metastases because of microscopic cancer focus. Another possible interpretation is that sampling error might be occurred and it is possible that metastatic PLANs with surgical excision was not the same aspirated lymph node. When the patients are subject to inadequate specimens, the oncologists must combine clinical data and propose US-FNAC once more. Although the treatment 
decisions based on PET/CT imaging(15) in some patients, it is recommended to obtain pathological evidence before chemoradiotherapy to reduce unnecessary medical disputes.

The strengths of this study contain the relatively many cervical cancer patients with PLANs enlargement,concerning a high proportion with small PLANs in size. In addition, our study is the first article that evaluates some factors influencing diagnostic yield in large samples and highlights independent predictor of diagnostic accuracy by multivariate analysis. What is more, this study investigates the role of the US-FNAC technique in clinical decision making.

The study has the following weaknesses. To begin with, this study is retrospective and has selectivity bias. Next, the diagnosis and treatment of some patients are based on clinical follow up and imaging data. Once more, this is a single-center study and the technical level is different. Ultimately, this study does not make an overall survival analysis, which is the focus of the following research.

In conclusion, US-FNAC is a safe, widely and conveniently available technique. The diagnostic accuracy of this technique is high and the main influencing factor is the experience of the puncturer. Therefore, it is suggested that the operators should accept formal standardized training and qualification certification. No false positives and low false negatives are conducive to clinical decision-making. US-FNAC technique can be added to standard pretherapy examinations for enlarged para-aortic lymph nodes in cervical cancer.

\section{Abbreviations}

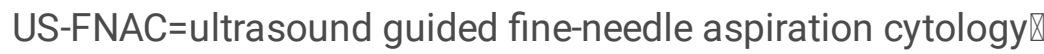

PALN=para-aortic lymph node囚DW-MRI=diffusion-weighted MR imaging

PET/CT=positron emission tomography and computed tomography

IMA=inferior mesenteric artery

\section{Declarations}

\section{Ethics approval and consent to participate}

This study was approved by the Ethics Committee of Zhejiang Cancer Hospital and were in accordance with the ethical standards of the 1964 Helsinki Declaration. The need for informed consent from all patients was waived due to the study's retrospective.

\section{Consent for publication}

Not applicable.

\section{Competing interests}

No potential conflict of interest was reported by the authors.

\section{Availability of data and materials}


The datasets used and/or analyzed during the current study are not publicly available due privacy rules but are available from the first author on reasonable request at the following email address liujp85@zjcc.org.cn

\section{Acknowledgements}

Not applicable.

\section{Funding}

This study was supported in part by the International Cooperation Project of Zhejiang Basic Public Technology Research Program囚LGJ20H010001ه囚Projects of Zhejiang Province Medical and Health Science and Technology Plan (2017KY256) \Project of Zhejiang Basic Public Technology Research Program『LSD19H180001】.

\section{Author information}

1. Department of Ultrasound $₫$ Cancer Hospital of the University of Chinese Academy of Sciences $₫$ Zhejiang Cancer

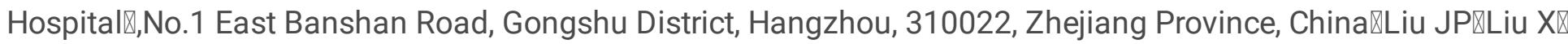
Xu D, Wang LJUQ

2. Department of Pathology $\llbracket$ Cancer Hospital of the University of Chinese Academy of Sciences $₫$ Zhejiang Cancer

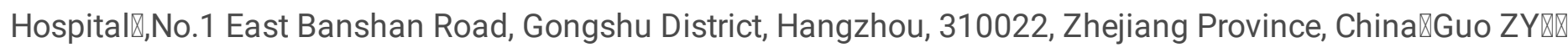

3. Department of Gynecologic Oncology $\$ Cancer Hospital of the University of Chinese Academy of Sciences \Zhejiang Cancer Hospital『,No.1 East Banshan Road, Gongshu District, Hangzhou, 310022, Zhejiang Province,

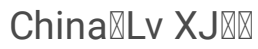

4. Zhejiang Key Laboratory of the Diagnosis \& Treatment Technology on Thoracic Oncology,No.1 East Banshan Road, Gongshu District, Hangzhou, 310022, Zhejiang Province, China『Mao WM区

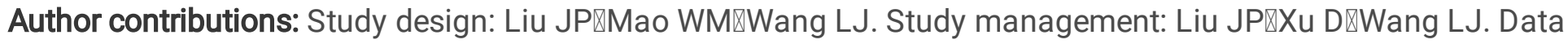

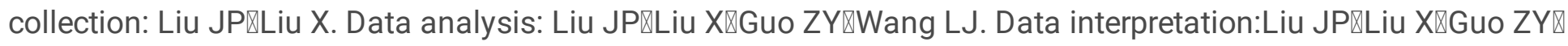
Lv XJ. Writing: Liu JP®Liu X『Guo ZY®Lv XJ.

Figures: Liu JP囚Liu X囚Lv XJ. All authors were involved in reviewing each manuscript draft and approving the final submitted version. Liu JP and Mao WM are responsible for the overall content as guarantors.

\section{References}

1. Shim SH, Kim DY, Lee SJ, et al. Prediction model for para-aortic lymph node metastasis in patients with locally advanced cervical cancer. Gynecol Oncol 2017;144(1):40-45.

2. Hwang L , Bailey A, Lea J , et al. Para-aortic nodal metastases in cervical cancer: a blind spot in the International Federation of Gynecology and Obstetrics staging system: current diagnosis and management. Future Oncology 2015, 11(2):309-322.

3. Eisenhauer EA, Terasse P, Bogaerts J, et al. New response evaluation criteria in solid tumours: revised RECIST guideline (version 1.1). Eur J Cancer 2009;45(2):228-247. 
4. Yang WT, Lam WM, Yu MY, et al. Comparison of dynamic helical CT and dynamic MR imaging in the evaluation of pelvic lymph nodes in cervical carcinoma. AJR Am J Roentgenol 2000;175(3):759-766.

5. Liu, Bin, Gao, Sujuan, Li, Shuofeng. A Comprehensive Comparison of CT, MRI, Positron Emission Tomography or Positron Emission Tomography/CT, and Diffusion Weighted Imaging-MRI for Detecting the Lymph Nodes Metastases in Patients with Cervical Cancer: A Meta-Analysis Based on 67 Studies. Gynecol Obstet Invest 2017;82(3):209-222

6. Atri M, Zhang Z, Dehdashti F, et al. Utility of PET-CT to evaluate retroperitoneal lymph node metastasis in advanced cervical cancer: results of ACRIN6671/GOG0233 trial. Gynecol Oncol 2016;142(3):413-419.

7. Gee MS, Atri M, Bandos Al, et al. Identifcation of distant metastatic disease in uterine cervical and endometrial cancers with FDG PET/CT: analysis from the ACRIN 6671/GOG 0233 Multicenter Trial. Radiology 2018;287(1):176-184.

8. Brunette $L \mathrm{~L}$, Bonyadlou S , Ji L , et al. Predictive Value of FDG PET/CT to Detect Lymph Node Metastases in Cervical Cancer. Clinical nuclear medicine 2018, 43(11):793-801 .

9. Achouri A, Huchon C, Bats AS, Bensaid C, Nos C, Lécuru F. Complications of lymphadenectomy for gynecologic cancer. Eur J Surg Oncol 2013;39(1):81-86.

10. De Filippo M, Saba L, Rossi E, et al. Curved needles in CT-guided fine needle biopsies of abdominal and retroperitoneal small lesions. J Vasc Interv Radiol 2015; 38(6):1611-1616.

11. Haibo, Shao, Colin, et al. CT-Guided Percutaneous Needle Biopsy of Retroperitoneal and Pelvic Lymphadenopathy: Assessment of Technique, Diagnostic Yield, and Clinical Value. J Vasc Interv Radiol 2018 ;29(10):1429-1436.

12. Meme! OS, Dodd GD III, Esola CC. Efficacy of sonography as a guidance technique for biopsy of abdominal, pelvic and retroperitoneal lymph nodes. AJR 1996;167(4):957-962.

13. Fisher AJ, Paulson EK, Sheafor DS, et al. Small lymph nodes of the abdomen, pelvis, and retroperitoneum: usefulness of sonographically guided biopsy. Radiology 1997; 205(1):185-190.

14. Smith EH. Complications of percutaneous abdominal fine-needle biopsy. Radiology 1991; 178(1):253-258.

15. National Comprehensive Cancer Network. Cervical cancer clinical practice guidelines in oncology.(v.l. 2020). https://www.nccn.org/professionals/physician_gls/pdf/cervical.pdf.

16. Screaton NJ , Berman LH, Grant JW. Head and Neck Lymphadenopathy: Evaluation with US-guided CuttingNeedle Biopsy. Radiology 2002;224(1):75-81.

17. Kurita A, Kodama Y, Nakamoto Y, et al. Impact of EUS-FNA for preoperative para-aortic lymph node staging in patients with pancreatobiliary cancer. Gastrointestinal Endoscopy 2016 ;84(3):467-475.

\section{Tables}

Table 1 Comparision of demographics and lesion distribution among the five groups. 


\begin{tabular}{|c|c|c|c|c|c|c|c|}
\hline $\begin{array}{l}\text { Characteristics } \\
\text { Group }\end{array}$ & $\begin{array}{l}\text { Total } \\
(n=92)\end{array}$ & $\begin{array}{l}\text { Malignant } \\
(n=62)\end{array}$ & $\begin{array}{l}\text { Suspicious } \\
(n=11)\end{array}$ & $\begin{array}{l}\text { Undetermined } \\
(n=5)\end{array}$ & $\begin{array}{l}\text { Benign } \\
(n=10)\end{array}$ & $\begin{array}{l}\text { Inadequate } \\
(n=4)\end{array}$ & $\begin{array}{l}P \\
\text { value }\end{array}$ \\
\hline Age[ $[ \pm s]$ (year) & $51.5 \pm 10.8$ & $50.9 \pm 9.4$ & $58.6 \pm 15.5$ & $52.8 \pm 15.5$ & $46.0 \pm 8.8$ & $54.5 \pm 7.4$ & 0.087 \\
\hline $\begin{array}{l}\text { Body mass } \\
\text { index }\end{array}$ & $22.0 \pm 3.4$ & $21.7 \pm 3.4$ & $23.7 \pm 5.0$ & $22.9 \pm 1.6$ & $21.5 \pm 2.1$ & $20.8 \pm 1.6$ & 0.302 \\
\hline $\begin{array}{l}\text { Lesion } \\
\text { size(mm) }\end{array}$ & $17.1 \pm 4.0$ & $17.6 \pm 3.8$ & $18.0 \pm 4.7$ & $16.0 \pm 4.0$ & $14.6 \pm 3.1$ & $13.8 \pm 3.2$ & 0.061 \\
\hline Nodal number & & & & & & & 1.000 \\
\hline solitary & $\begin{array}{l}13 \\
\square 14.1 \% \bigotimes\end{array}$ & $7 \rrbracket 7.6 \% \rrbracket$ & $1 \otimes 1.1 \% \bigotimes$ & $0 \otimes 0.0 \% \rrbracket$ & $4 \llbracket 4.3 \% \bigotimes$ & $1 \otimes 1.1 \% \bigotimes$ & \\
\hline multiple & $\begin{array}{l}79 \\
\varangle 85.9 \% \rrbracket\end{array}$ & $\begin{array}{l}55 \\
\otimes 59.8 \% \bigotimes\end{array}$ & $10 \rrbracket 10.9 \% \rrbracket$ & $5 \rrbracket 5.4 \% \rrbracket$ & $6 \rrbracket 6.5 \% \rrbracket$ & $3 \llbracket 3.3 \% \bigotimes$ & \\
\hline \multicolumn{8}{|l|}{ Location } \\
\hline \multicolumn{8}{|l|}{$\begin{array}{l}\text { Left } \\
\text { Paraaortic }\end{array}$} \\
\hline $\begin{array}{l}\text { Above the } \\
\text { IMA(囚) }\end{array}$ & $\begin{array}{l}27 \\
\otimes 29.3 \% \bigotimes\end{array}$ & $\begin{array}{l}18 \\
\otimes 19.5 \% \bigotimes\end{array}$ & $5 \otimes 5.4 \% \bigotimes$ & $1 \otimes 1.1 \% \square$ & $0 \rrbracket 0.0 \% \bigotimes$ & $3 \rrbracket 3.3 \% \rrbracket$ & 0.670 \\
\hline $\begin{array}{l}\text { Below the } \\
\text { IMA(囚) }\end{array}$ & $\begin{array}{l}28 \\
\varangle 30.5 \% \rrbracket\end{array}$ & 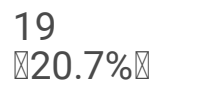 & $4 \llbracket 4.3 \% \rrbracket$ & $1 \otimes 1.1 \% \rrbracket$ & $3 \llbracket 3.3 \% \bigotimes$ & $1 \otimes 1.1 \% \bigotimes$ & \\
\hline \multicolumn{8}{|l|}{$\begin{array}{l}\text { Right } \\
\text { Paraaortic }\end{array}$} \\
\hline $\begin{array}{l}\text { Below the } \\
\text { IMA( }(\mathbb{B})\end{array}$ & $\begin{array}{l}29 \\
\varangle 31.5 \% \bigotimes\end{array}$ & 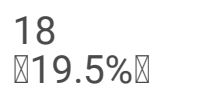 & $2 \rrbracket 2.2 \% \rrbracket$ & $3 \rrbracket 3.3 \% \rrbracket$ & $6 \rrbracket 6.5 \% \bigotimes$ & $0 \rrbracket 0.0 \% \rrbracket$ & 1.000 \\
\hline $\begin{array}{l}\text { Above the } \\
\text { IMA(囚) }\end{array}$ & $8 \llbracket 8.7 \% \rrbracket$ & $7 \rrbracket 7.6 \% \rrbracket$ & $0 \rrbracket 0.0 \% \rrbracket$ & $0 \rrbracket 0.0 \% \rrbracket$ & $1 \rrbracket 1.1 \% \bigotimes$ & $0 \rrbracket 0.0 \% \bigotimes$ & \\
\hline \multicolumn{8}{|l|}{ Needle gauge } \\
\hline 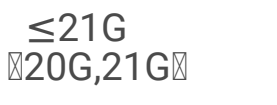 & $\begin{array}{l}60 \\
\otimes 65.2 \% \bigotimes\end{array}$ & $\begin{array}{l}40 \\
\varangle 43.5 \% \rrbracket\end{array}$ & $8 \llbracket 8.7 \% \rrbracket$ & $4 \llbracket 4.3 \% \rrbracket$ & $6 \rrbracket 6.5 \% \rrbracket$ & $2 \llbracket 2.2 \% \bigotimes$ & 1.000 \\
\hline 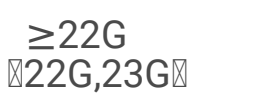 & $\begin{array}{l}32 \\
\varangle 34.8 \% \rrbracket\end{array}$ & $\begin{array}{l}22 \\
\otimes 23.9 \% \rrbracket\end{array}$ & $3 \llbracket 3.3 \% \rrbracket$ & $1 \otimes 1.1 \% \bigotimes$ & $4 \llbracket 4.3 \% \rrbracket$ & $2 \llbracket 2.2 \% \bigotimes$ & \\
\hline Cell block & & & & & & & 0.483 \\
\hline Yes & $\begin{array}{l}31 \\
\bigotimes 33.7 \% \bigotimes\end{array}$ & 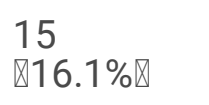 & $9 \varangle 9.9 \% \rrbracket$ & $1 \otimes 1.1 \% \rrbracket$ & $3 \llbracket 3.3 \% \rrbracket$ & $3 \rrbracket 3.3 \% \rrbracket$ & \\
\hline No & $\begin{array}{l}61 \\
\varangle 66.3 \% \bigotimes\end{array}$ & $\begin{array}{l}47 \\
\varangle 51.1 \% \bigotimes\end{array}$ & $2 \llbracket 2.2 \% \rrbracket$ & $4 \llbracket 4.3 \% \rrbracket$ & $7 区 7.6 \% \bigotimes$ & $1 \otimes 1.1 \% \bigotimes$ & \\
\hline $\begin{array}{l}\text { Final } \\
\text { diagnosis }\end{array}$ & & & & & & & 0.110 \\
\hline Malignant & $\begin{array}{l}84 \\
\square 91.3 \% \square\end{array}$ & $\begin{array}{l}62 \\
\square 67.4 \% \bigotimes\end{array}$ & $11 \otimes 11.9 \% \rrbracket$ & $5 \rrbracket 5.4 \% \bigotimes$ & $3 \llbracket 3.3 \% \bigotimes$ & $3 \llbracket 3.3 \% \rrbracket$ & \\
\hline benign & $8 \rrbracket 8.7 \% \rrbracket$ & $0 \otimes 0.0 \% \bigotimes$ & $0 \otimes 0.0 \% \bigotimes$ & $0 \otimes 0.0 \% \rrbracket$ & $7 ه 7.6 \% \rrbracket$ & $1 \rrbracket 1.1 \% \rrbracket$ & \\
\hline
\end{tabular}




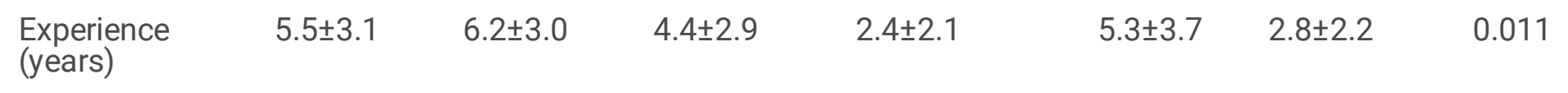

Note.Experience refers to the years of engaging in interventional ultrasound.

Table 2هThe diagnostic categorizations of initial US-FNAC in PLANs and final diagnosis

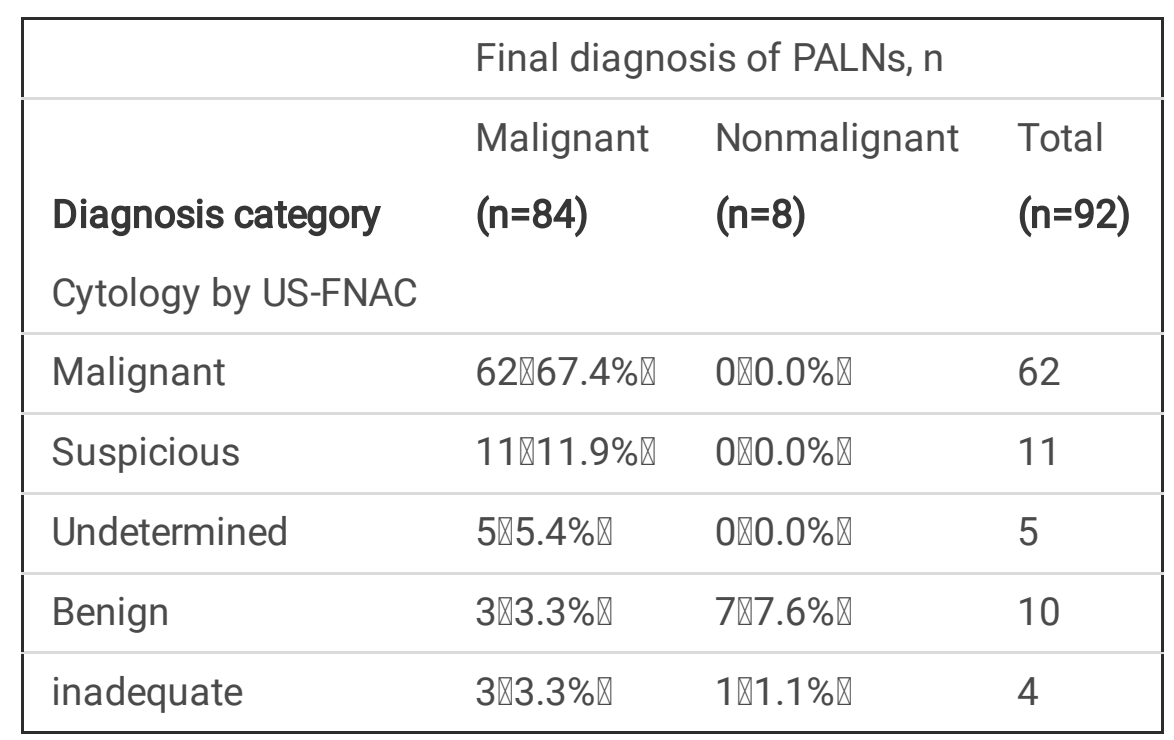

Note.-Final diagnoses were confirmed by at least two experts, including radio-oncologists, imaging specialists, and pathologists(15). US-FNAC=ultrasound guided fine-needle aspiration cytology $\mathbb{P A L N s}=$ para-aortic lymph nodes

Table 3囚The diagnostic performance of US-FNAC for PLANs in cervical cancer

\begin{tabular}{|llll|}
\hline Diagnostic index & Inclusion of Undetermined & Exclusion of Undetermined & $P$ value \\
\hline Sensitivity, \%(n) & $90.1 \%(73 / 81)$ & $96.1 \%(73 / 76)$ & 0.146 \\
\hline Specificity, \%(n) & $100 \%(7 / 7)$ & $100 \%(7 / 7)$ & 1.000 \\
\hline PPV, \%(n) & $100 \%(73 / 73)$ & $100 \%(73 / 73)$ & 1.000 \\
\hline NPV, \%(n) & $46.7 \%(7 / 15)$ & $70.0 \%(7 / 10)$ & 0.250 \\
Accuracy, \%(n) & $90.9 \%(80 / 88)$ & $96.4 \%(80 / 83)$ & 0.145 \\
\hline
\end{tabular}

PPV,Positive predictive value; NPV,Negative predictive value

Table $4 \llbracket$ Variate analysis of the factors influencing diagnostic yield 


\begin{tabular}{|c|c|c|c|c|c|c|}
\hline \multirow[t]{2}{*}{ Variables } & \multirow{2}{*}{$\begin{array}{l}\text { Correct diagnosis } \\
(n=81)\end{array}$} & \multirow{2}{*}{$\begin{array}{l}\text { Wrong } \\
\text { Diagnosis } \\
(n=11)\end{array}$} & \multirow{2}{*}{$\begin{array}{l}\text { Univariate } \\
\text { analysis } \\
P \text { value }\end{array}$} & \multicolumn{2}{|c|}{ Multivariate analysis } & \multirow[b]{2}{*}{$P$ value } \\
\hline & & & & OR & $95 \% \mathrm{Cl}$ & \\
\hline Age[ $\pm s \bigotimes$ range $\bigotimes]$ (year) & $51.3 \pm 10.8$ & $53.1 \pm 11.0$ & 0.614 & 1.039 & $0.96-1.124$ & 0.344 \\
\hline Nodal size, mm & & & 0.322 & 0.333 & $0.073-1.510$ & 0.154 \\
\hline$>15 \mathrm{~mm}$ & 52 & 5 & & & & \\
\hline$\leq 15 \mathrm{~mm}$ & 29 & 6 & & & & \\
\hline Nodal number & & & 0.353 & -- & -- & -- \\
\hline multiple & 68 & 11 & & & & \\
\hline solitary & 13 & 0 & & & & \\
\hline Nodal location & & & & 0.553 & $0.106-2.877$ & 0.481 \\
\hline Right Paraaortic & 49 & 5 & 0.352 & & & \\
\hline Left Paraaortic & 32 & 6 & & & & \\
\hline Needle gauge & & & 0.182 & 1.090 & $0.235-5.049$ & 0.913 \\
\hline$\leq 21 \mathrm{G}$ & 26 & 6 & & & & \\
\hline$\geq 22 \mathrm{G}$ & 55 & 5 & & & & \\
\hline Body mass index $\triangle \mathrm{BM} \mathbb{}$ & & & 0.446 & 0.187 & $0.015-2.392$ & 0.197 \\
\hline$\leq 24$ & 61 & 10 & & & & \\
\hline$>24$ & 20 & 1 & & & & \\
\hline Cell block & & & 0.498 & 1.470 & $0.212-10.20$ & 0.697 \\
\hline Yes & 26 & 5 & & & & \\
\hline No & 55 & 6 & & & & \\
\hline Nature of lymph node & & & 0.589 & -- & -- & -- \\
\hline Malignant & 73 & 11 & & & & \\
\hline Benign & 8 & 0 & & & & \\
\hline Experience (years) & & & 0.008 & 0.077 & $0.007-0.793$ & 0.031 \\
\hline$\leq 5$ & 37 & 10 & & & & \\
\hline$>5$ & 44 & 1 & & & & \\
\hline Period & & & 0.197 & 2.501 & $0.372-16.83$ & 0.346 \\
\hline $2010-2015$ & 43 & 3 & & & & \\
\hline $2016-2018$ & 38 & 8 & & & & \\
\hline
\end{tabular}




\section{Figures}

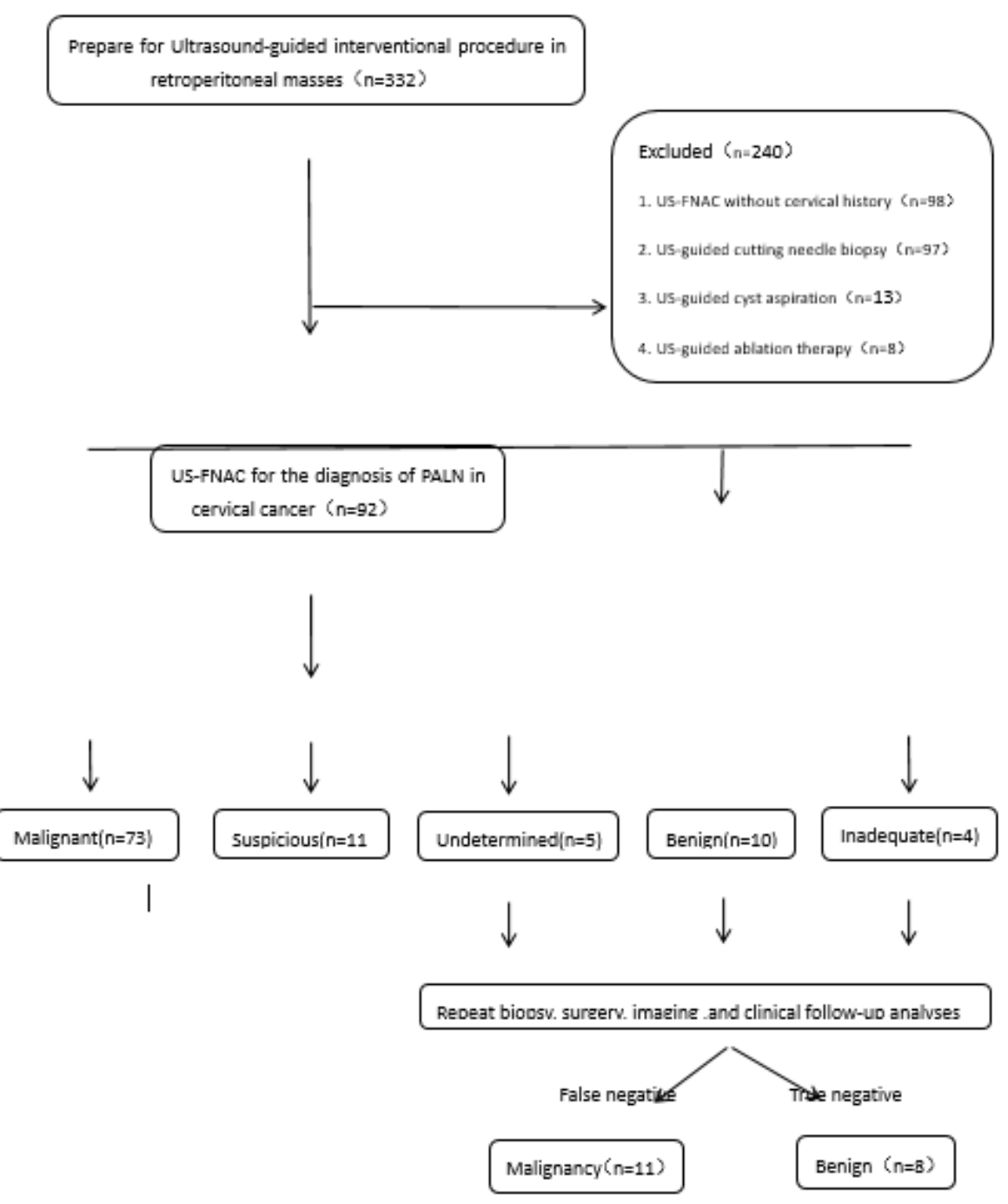

\section{Figure 1}

Flow chart of US-FNAC from 2010-2018 for diagnosis of PALN in cervical cancer. 


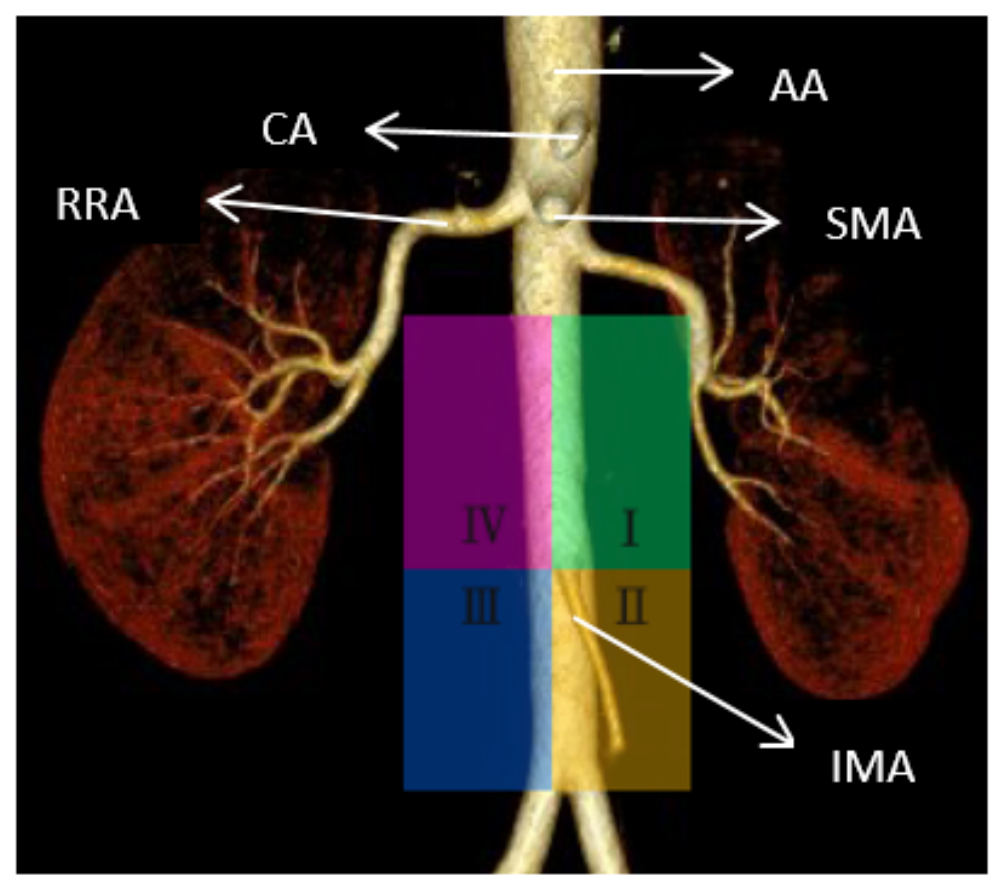

Figure 2

$\mathrm{SMA}=$ Superior mesenteric artery $\mathrm{AA}=\mathrm{Abdominal}$ aorta RRA=Right renal artery IMA=Inferior mesenteric artery $\mathrm{CA}=$ Celiac axis Schematic diagram of the anatomical distribution of the para-aortic lymph nodes. 

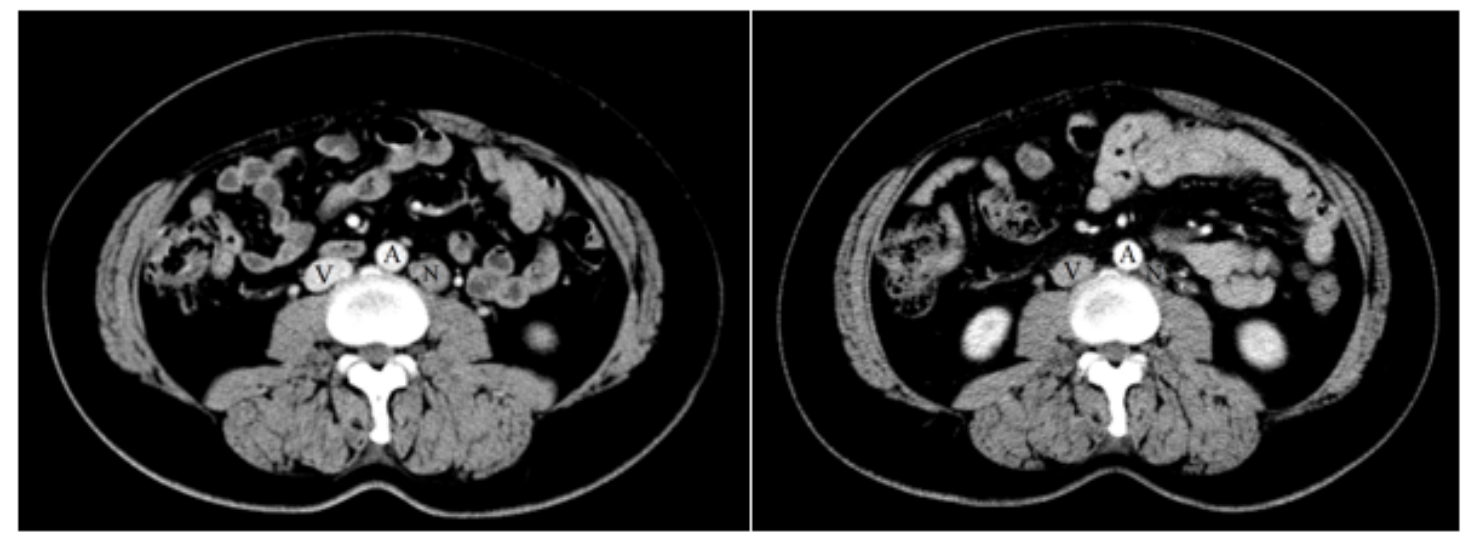

a.

b.
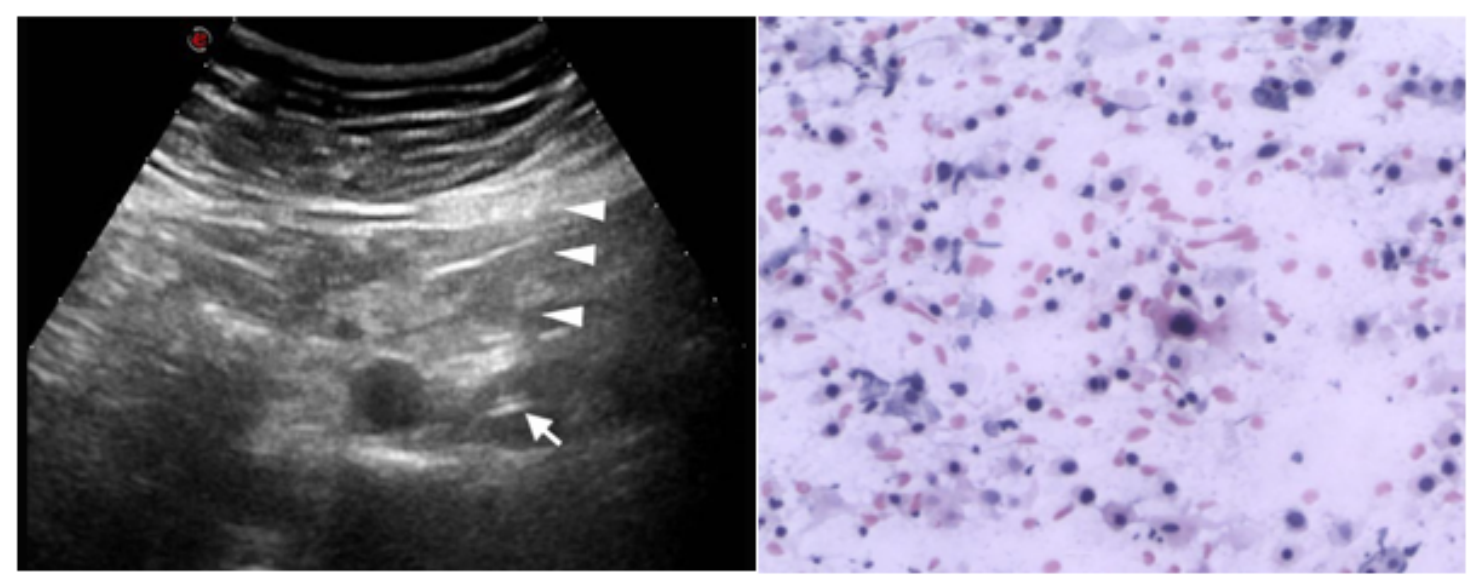

c.

d.

\section{Figure 3}

$\mathrm{A}=$ aorta; $\mathrm{V}=$ Inferior vena cava; $\mathrm{N}=$ Lymph node US-FNAC and therapeutical effect of a para-aortic lymph node . (a) a 49-year-old woman with metastatic squamous carcinoma from cervical cancer presents with a $16 \mathrm{~mm}$ Lymph node located left paraaortic region above the inferior mesenteric artery( $\nabla)$. (b) After 5 months of radiotherapy, the size of lymph nodes are significantly reduced. (c) Needle tip (arrow) is clearly defined as being within the Lymph node and conventional US displays the pass of the puncture needle through the mesentery or bowels(arrowhead). (d ) The FNAC results was diagnosed as metastatic squamous cell carcinoma with scattered atypical cells, necrosis and cytokeratinization (HE stain,×20) . 


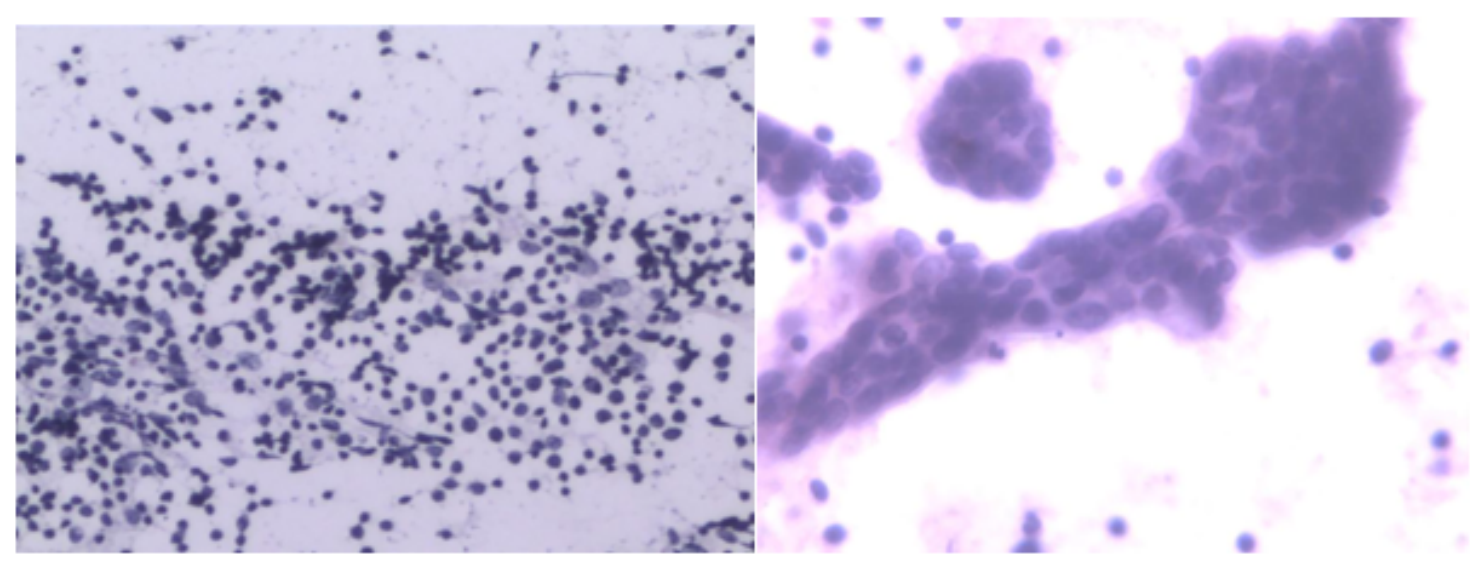

a.

b.

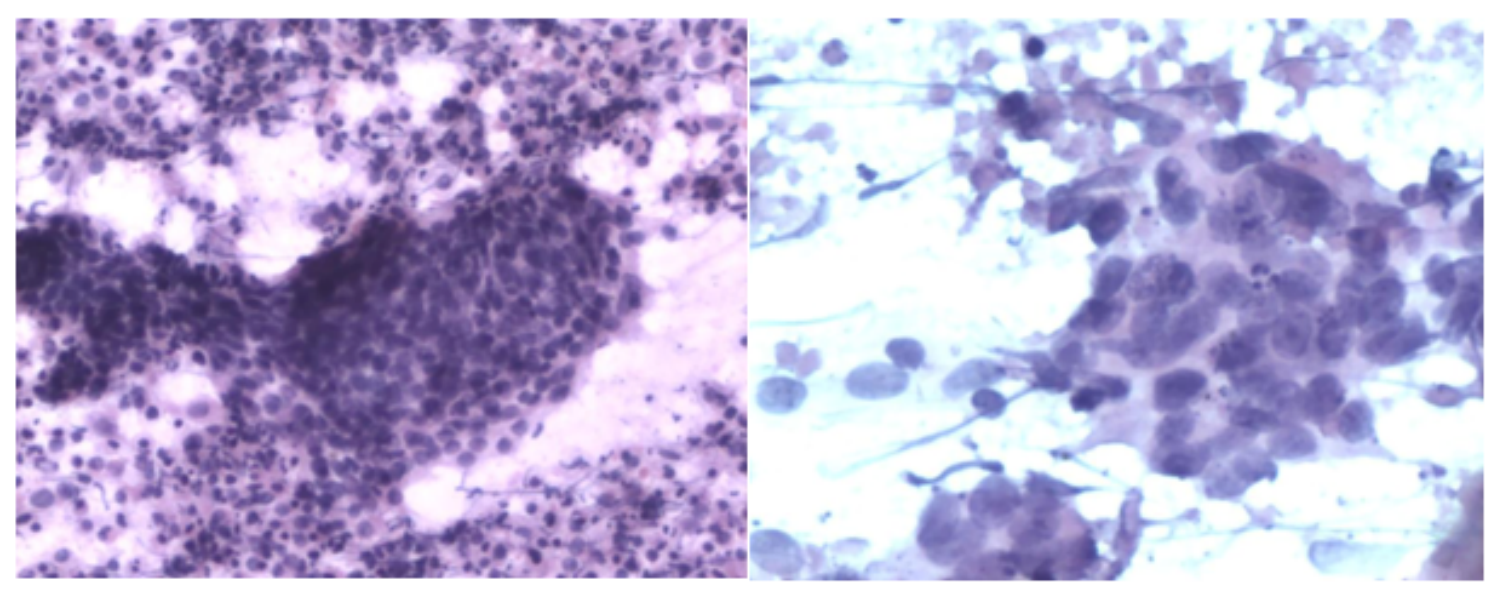

c.

d.

\section{Figure 4}

The cytological morphology diagnosis of US-FNAC in para-aortic lymph nodes. (a) Cytology smear showing scattered lymphocytes and histiocytes with mixed morphology is reported as benign results (HE stain, $\times 10)$. (b) Cytology smear showing glandular clusters with atypical epithelial cells scattered in the lymphocyte background is diagnosed as metastatic adenocarcinoma (HE stain,×20) . (c) In the degenerated necrotic lymphoid tissue, there are clusters of atypical epithelial cells arranged in whirlpool, showing keratinization tendency, which is regarded as metastatic squamous cell carcinoma (HE stain,×20) . (d) In a large necrotic background, poorly differentiated cancer cells were seen in clusters, with obvious nucleoli and unclear differentiation tendency, which is considered as metastatic undifferentiated carcinoma (HE stain, $\times 40)$.

\section{Supplementary Files}

This is a list of supplementary files associated with this preprint. Click to download.

- Videoscaption.docx

- movie1.avi

- movie2.avi 